\title{
Contrast-Enhanced Intraoperative Ultrasonography with Kupffer Phase May Change Treatment Strategy of Metastatic Liver Tumors - A Single-Centre Prospective Study
}

\author{
Chen $\mathrm{Li}^{1}$ \\ Yuan Liu' \\ Jingyong $\mathrm{Xu}$ (iD $^{2}$ \\ Jinghai Song ${ }^{2}$ \\ Mingxiao $\mathrm{Wu}^{\prime}$ \\ Jian Chen ${ }^{2}$
}

'Department of Ultrasonography, Beijing Hospital, National Centre of Gerontology, Institute of Geriatric Medicine, Chinese Academy of Medical Sciences, Beijing, I00730, People's Republic of China; ${ }^{2}$ Department of General Surgery, Beijing Hospital, National Centre of Gerontology, Institute of Geriatric Medicine, Chinese Academy of Medical Sciences, Beijing, 100730, People's Republic of China
Correspondence: Jingyong $\mathrm{Xu}$ Department of General Surgery, Beijing Hospital, No. I, Dahua Road, Dongdan, Beijing, 100730, People's Republic of China

Tel +86138I035I797

Email xujingyong@bjhmoh.cn
Aim: To compare the diagnostic performance of contrast-enhanced intraoperative ultrasonography (CE-IOUS) with Kupffer phase in metastatic liver tumours.

Methods: Twenty-seven consecutive patients with liver metastasis were prospectively recruited from November 2019 to July 2020 in the Department of HPB, Beijing Hospital. MRI and Contrast Enhanced Ultrasonography (CEUS) were obtained preoperatively, and the diagnosis was made by radiologists independently and blindly. Intraoperative ultrasonography (IOUS) and CE-IOUS with Sonazoid were done by the same sophisticated surgeon and sonographer and Kupffer phase was used to detect lesions. The sensitivity and specificity to detect lesions were compared between different radiologic methods. Then, the changes in treatment strategy due to CE-IOUS with Sonazoid were analysed.

Results: Twenty-seven patients were included. In MRI, 91 lesions were detected with sensitivity $93.3 \%$ (70/75) and specificity $68.8 \%$ (11/16). In CEUS, it was 97.1\% (68/70) and $86.7 \%(13 / 15)$ in 85 lesions. Meanwhile, in the Kupffer phase in CE-IOUS, 99 lesions were found and 8 new lesions were discovered in 7 cases, with sensitivity $97.5 \%(80 / 82)$ and specificity $94.1 \%(16 / 17)$. The four imaging methods showed no statistic significance in sensitivity and specificity in detecting lesions (Cochran's Q 10.825, $P=0.055$ ). Treatment strategies were altered in 7 patients, 6 achieved R0 resection or ablation, and 1 patient changed from planned R0 resection to palliative surgery.

Conclusion: CE-IOUS may play a similar or even better role than other radiological methods in diagnosing liver metastasis. The CE-IOUS using Sonazoid demonstrated a high sensitivity and specificity for finding occult metastases intraoperatively and changing the treatment strategy.

Keywords: contrast-enhanced intraoperative ultrasonography, Kupffer phase, liver metastasis, Sonazoid

\section{Introduction}

Intraoperative ultrasound (IOUS) is considered as an intraoperative method to determine the margin of lesions and to detect preoperatively undiscovered tumors. $^{1,2}$ The use of IOUS for liver surgery is effective for detecting occult or additional nodules. $^{3-5}$ Meanwhile, contrast-enhanced IOUS (CE-IOUS) with Sonazoid has improved the detection and identification of malignant liver lesions and subsequently changed the surgical plan. ${ }^{6,7}$ 
Sonazoid (perfluorobutane, GE Healthcare, Oslo, Norway) provides a parenchyma-specific contrast image based on its accumulation in the Kupffer cells in the liver. ${ }^{8}$ It presents with a late Kupffer-phase image with a long duration for scanning the whole liver of more than 1 hour. The Kupffer phase by CE-IOUS is more sensitive than IOUS and MRI to detect small malignant lesions less than $1 \mathrm{~cm}^{9,10}$

The aim of our study was to estimate the capability of IOUS and CE-IOUS to detect occult liver lesions in liver metastasis surgery.

\section{Materials and Methods}

Twenty-seven Consecutive patients with liver metastasis were prospectively recruited from November 2019 to July 2020 in the Department of General Surgery, Beijing Hospital. These patients included 20 males and 7 females and had a mean age of 59.6 (range, 33-83). The primary lesion was located in the rectum in 13 cases, breast in 4 , pancreas in 3 , colon in 4 , stomach in 3 , duodenum in 1 .

This study was approved by the Ethics Committee regarding Human Research of Beijing Hospital. All experiments were performed following the relevant guidelines and regulations of the Institutional Ethics Committee and the Declaration of Helsinki, 1975. All patients provided written informed consent.

\section{CEUS Techniques}

All patients underwent conventional US, CEUS with Sonazoid. All examinations were performed by two sonographers using an Aplio 500 (Canon, Honshu, Japan) with a convex probe $(6 \mathrm{C} 1,1-6 \mathrm{MHz})$ and a linear probe $(11 \mathrm{~L} 4$, 4-11 MHz). The mechanical index (MI) was set to be 0.2 . The patients received a bolus intravenous injection of Sonazoid $(0.015 \mathrm{~mL} / \mathrm{kg}$ body weight $)$ through a peripheral venous line, followed by $5 \mathrm{~mL}$ of normal saline flush. Immediately after the administration of Sonazoid, the portal veins, hepatic veins, and the normal liver parenchyma were uniformly enhanced during an early vascular-phase image lasting $3 \mathrm{~min}$. Approximately $10 \mathrm{~min}$ after the injection, the liver was scanned again to observe the post-vascular phase image (Kupffer phase). In all patients, including those with no lesion visualized on US, the whole liver was observed in the Kupffer phase, and the presence of defects was evaluated. Visualized defects were examined using defect reperfusion imaging, in which liver metastasis would be observed. ${ }^{11}$ Diagnostic features of liver metastases were early washout in the portal venous phase along with hypo-enhancements during portal venous and Kupffer phases.

\section{MRI Protocols}

All patients underwent MRI exams on a 3.0-T whole-body scanner (Signa Pioneer, GE Healthcare, Milwaukee, USA). T1-weighted images (T1WI), T2-weighted images (T2WI) and diffusion-weighted images (DWI) were obtained. Images were obtained in the transverse plane. Dynamic MRI was acquired before and at 20 and 60 seconds and 2, 5, 10 minutes after injection of gadolinium diamine. The contrast medium $(0.2 \mathrm{~mL} / \mathrm{kg}$ of body weight $)$ was injected intravenously and flushed with $20 \mathrm{~mL}$ of saline using a power injector.

The final diagnosis and identification of liver metastasis were made by pathological examination using resected specimens and biopsy or follow-up imaging modalities (changes of size).

\section{IOUS and CE-IOUS}

Examination of IOUS and CE-IOUS was performed using a HITACHI ALOKA (ARIETTA 70, Tokyo, Japan), laparoscopic probe (L44LA, 2-13 MHz) and microconvex probe (C42T, 3-10 MHz). CE-IOUS was performed under the pulse inversion harmonic (PIH) imaging capability. A bolus intravenous injection of Sonazoid $[0.015 \mathrm{~mL} / \mathrm{kg}$ body weight] was performed via the central venous line followed by $5 \mathrm{~mL}$ of normal saline flush. The injection could be repeated.

During the intra-operatory evaluation, IOUS was performed to search for new nodules. And then, CE-IOUS was carried out both for lesion characterization and for new nodule detection. If new lesions were detected in IOUS, CE-IOUS was performed in vascular phases to distinguish between benign and malignant lesions. After 10 mins, the whole liver was scanned again to observe the post-vascular image (Kupffer image). Kupffer defects detected in the Kupffer phase and defect reperfusion imaging are useful to distinguish true metastatic lesions from other types of tumors.

MRI and CEUS were obtained preoperatively, and the diagnosis was made by 2 radiologists independently and blindly. All included patients were discussed by the multidisciplinary team (MDT). IOUS and CE-IOUS with Sonazoid were done by the same sophisticated surgeon and sonographer. Neither the radiologist nor the surgeon performing the IOUS was blinded to the findings of any of the investigations. The imaging of MRI, IOUS and CE- 
IOUS were compared. The interval between the preoperative imaging and intraoperative findings should be less than 15 days. The surgical staging or management of the disease noted the changes.

\section{Statistical Analysis}

Statistical analysis was performed using MedCalc 19.3.1 (MedCalc Software Ltd) software. In order to verify any differences in the detection of liver metastasis capability among the four imaging methods, we used the nonparametric Cochran Q-test.

The sensitivity, specificity, accuracy, positive predictive value (PPV), negative predictive value (NPV), 95\% confidence interval $(95 \% \mathrm{CI})$ and Youden index (Y) were calculated by use of standard statistical formulae. Number needed to diagnose (NND), predictive summary index (PSI), number needed to predict (NNM) and number needed to misdiagnose (NNM) which can be calculated accordingly. A $p$ value of less than 0.05 was considered statistically significant.

\section{Results}

There were 99 lesions detected in the 27 patients. Of these lesions, 82 were diagnosed as metastases and 17 as nonmetastasis, with 88 diagnosed pathologically and 11 diagnosed according to course observation of at least 6 months using imaging modalities. The 17 non-metastasis lesions were 3 hemangiomas, 1 liver cyst, 2 focal nodular hyperplasias (FNH), 1 inflammatory lesion, 10 necrosis after treatment (Table 1).

The results of lesion-by-lesion analysis of sensitivity, specificity, PPV, NPV, accuracy, 95\% CI, Y, NND, PSI, NNP and NNM of each imaging method are reported in Table 2. The four imaging methods showed no statistic significance in sensitivity and specificity in detecting lesions (Cochran's Q 10.825, $P=0.055$ ).

Of the 27 patients, IOUS or CE-IOUS demonstrated additional or previously undetected liver lesions in 7 patients. A summary of the 7 patients is reported in Table 3. In these 7 patients, MRI had detected 36 lesions, preoperative CEUS detected 36 lesions, IOUS detected 42, and CE-IOUS detected 44 lesions. A total of 8 new lesions were diagnosed at CE-IOUS.

In the patient No.1 (Table 3), a $4 \mathrm{~mm}$ nodule at the Segment VIII was newly found by CE-IOUS during the Kupffer phase, MRI and CEUS did not detect this lesion because of volume effect. Another a $6 \mathrm{~mm}$ lesion at
Table I Characteristics of the Patients

\begin{tabular}{|c|c|}
\hline & All $(n=27)$ \\
\hline Age (years) (mean \pm SD) & $59.6 \pm 16.7$ \\
\hline \multicolumn{2}{|l|}{ Gender } \\
\hline Male/female & $20 / 7$ \\
\hline \multicolumn{2}{|l|}{ Sites of primary lesions } \\
\hline Rectum & 4 \\
\hline Colon & 13 \\
\hline Pancreas & 3 \\
\hline Breast & 4 \\
\hline Stomach & 2 \\
\hline Duodenum & I \\
\hline Liver tumors & 99 \\
\hline Liver metastases/ others & $82 / 17$ \\
\hline \multicolumn{2}{|l|}{ Others } \\
\hline Hepatic hemangioma & 3 \\
\hline Liver cyst & I \\
\hline $\mathrm{FNH}$ & 2 \\
\hline Inflammatory lesion & I \\
\hline Necrosis after treatment & 10 \\
\hline \multicolumn{2}{|l|}{ Confirmed diagnosis } \\
\hline Histopathological & 88 \\
\hline Various imaging modalities & 11 \\
\hline
\end{tabular}

Segment III was detected by both IOUS and CE-IOUS confirmed, while MRI and CEUS did not show this lesion. The two lesions were all resected and confirmed as metastatic nodules.

In the patient No.5, MRI and preoperative CEUS could not show the lesion with $6 \mathrm{~mm}$ in diameter at the Segment VI. During the surgery, IOUS did not show the lesion, while Kupffer phase in CE-IOUS showed a well-defined mass. The lesion at Segment VI was removed by laparoscopic radiofrequency ablation and pathologically verified as a metastasis. The patient had complete regression (Figure 1).

In the patient No.6, two additional hyperechoic lesions with 4-5 $\mathrm{mm}$ in diameter at Segment II and III were detected by IOUS and confirmed by CE-IOUS. The patient had five liver metastases and two benign lesions on both MRI and CEUS and two additional metastases on both IOUS and CE-IOUS and performed a palliative bypass instead of planned $\mathrm{R} 0$ resection.

In the patient No.14, a lesion at the Segment II was detected by MRI and considered suspicious for metastasis, 
Table 2 Lesion-by-Lesion Comparison Among Different Radiologic Imaging Methods

\begin{tabular}{|l|c|c|c|c|}
\hline Diagnosis Performance & MRI & CEUS & IOUS & CE-IOUS \\
\hline Sensitivity (\%) (95\% Cl) & $93.3(70 / 75)(85.1-97.8)$ & $97.1(68 / 70)(90.1-99.7)$ & $92.5(74 / 80)(84.4-97.2)$ & $97.5(80 / 82)(91.5-99.7)$ \\
PPV (\%) (95\% Cl) & $93.3(70 / 75)(87.1-96.7)$ & $97.1(68 / 70)(90.3-99.2)$ & $89.2(74 / 83)(83.9-92.8)$ & $98.8(80 / 81)(92.3-99.8)$ \\
Specificity (\%) (95\% Cl) & $68.8(1 / / 16)(41.3-89.0)$ & $86.7(13 / 15)(59.5-98.3)$ & $47.1(8 / 17)(23.0-72.2)$ & $94.1(16 / 17)(71.3-99.9)$ \\
NPV (\%) (95\% Cl) & $68.8(1 / / 16)(47.0-84.5)$ & $86.7(13 / 15)(62.0-96.3)$ & $57.1(8 / 14)(34.7-77.0)$ & $88.9(16 / 18)(66.9-96.9)$ \\
Accuracy (\%) & $89(81 / 91)$ & $95.3(81 / 85)$ & $84.5(82 / 97)$ & $97(96 / 99)$ \\
AUC (95\% Cl) & $0.810(0.7 / 5-0.885)$ & $0.919(0.839-0.967)$ & $0.698(0.596-0.787)$ & $0.958(0.898-0.988)$ \\
Y & 0.621 & 0.838 & 0.396 & 0.916 \\
NND & 1.610 & 1.193 & 2.525 & 1.092 \\
PSI & 0.621 & 0.838 & 0.463 & 0.877 \\
NNP & 1.610 & 1.193 & 2.160 & 1.140 \\
NNM & 9.091 & 21.277 & 6.452 & 33.333 \\
\hline
\end{tabular}

Abbreviations: CEUS, contrast-enhanced ultrasonography; IOUS, intraoperative ultrasonography, CE-IOUS, contrast-enhanced intraoperative ultrasonography, PPV, positive predictive value; NPV, negative predictive value; $95 \% \mathrm{Cl}, 95 \%$ confidence interval; Y, Youden index; NND, number needed to diagnose; PSI, predictive summary index; NNP, number needed to predict; NNM, number needed to misdiagnose.

while CEUS did not show this lesion. IOUS and CE-IOUS found the lesion confirmed the cyst. The findings changed planned management and cancelled radiofrequency ablation.

In the patient No.17, a hypoechoic nodule with $8 \mathrm{~mm}$ in diameter at Segment VIII was newly found by IOUS and confirmed by CE-IOUS although MRI and CEUS did not show this lesion. The lesion was removed by laparoscopic radiofrequency ablation and pathologically verified as a metastasis. The patient had complete regression.

In the patient No.23, MRI and preoperative CEUS could not show the lesion with $6 \mathrm{~mm}$ in diameter at the Segment V. During the surgery, the new lesion was detected by IOUS and confirmed by CE-IOUS. The lesion was resected and pathologically confirmed as a metastasis. A lesion at Segment IV was detected by MRI and CEUS, considered suspicious for metastasis. IOUS and CE-IOUS found that the lesion confirmed benign lesion. With intraoperative rapid frozen, the lesion was pathologically verified as necrosis after treatment. The finding changed planned management and cancelled resection.

In the patient No.24, a hypoechoic nodule with $9 \mathrm{~mm}$ in diameter at Segment IV was found by IOUS and confirmed by CE-IOUS. The patient had eight liver metastases and an additional metastasis on both IOUS and CE-IOUS and performed percutaneous or laparoscopic radiofrequency ablation and laparoscopic resection.

\section{Discussion}

IOUS is the most sensitive and accurate tool for the detection of additional liver nodules that are not detected by preoperative staging modalities used in liver metastases (met). CE-IOUS was more sensitive than IOUS to identify new lesions and to influence surgical management. In our study, 8 occult metastatic lesions were newly found in 7 of the 27 patients using CE-IOUS. These newly detected lesions changed the preoperatively planned surgery. Treatment strategies were altered in 7 patients, 6 achieved R0 management including 3 removed by additional hepatectomy and 3 removed by radiofrequency ablations, 1 patient changed from planned $\mathrm{R} 0$ resection to palliative surgery. Kupffer defects detected in the Kupffer phase by CE-IOUS are not always met, and defects reperfusion imaging is useful to distinguish true metastatic lesions from other types of tumors, such as cyst, hemangioma or FNH. ${ }^{12-14}$ In addition to the Kupffer defect, information on arterial vascularity, such as the reinjection method, increased the diagnostic accuracy to $100 \%$ even in deeply seated nodules. ${ }^{8}$ Four new lesions were discovered by IOUS and additional 4 occult lesions were detected by CEIOUS. Among 4 lesions discovered by IOUS, 2 lesions were verified as metastatic lesions by CE-IOUS, while IOUS was difficult to identify, 2 lesions were clearly diagnosed as metastatic lesions by IOUS. The additional 4 occult lesions detected by CE-IOUS were all diagnosed as metastatic lesions. Of 5 of 27 patients, CE-IOUS altered the diagnosis and detection of IOUS. The frequency of detecting occult nodules by CE-IOUS was more sensitive than that of IOUS and CE-IOUS seemed to be more accurate about the characterization of these lesions. ${ }^{15-18}$

Sonazoid is a new microbubble medium and is classified as a newly generation medium in which the perfluorocarbon gas has enough intravascular stability. ${ }^{19}$ The 


\begin{tabular}{|c|c|c|c|c|c|c|c|c|c|c|c|c|}
\hline 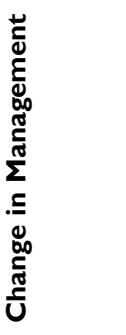 & & \multicolumn{2}{|l|}{ 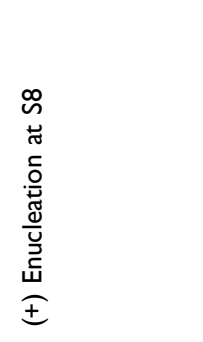 } & 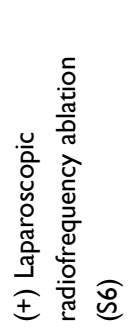 & \multicolumn{2}{|c|}{ 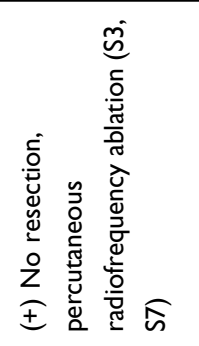 } & \multicolumn{2}{|l|}{ 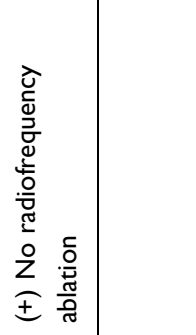 } & 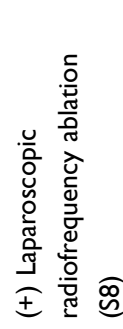 & 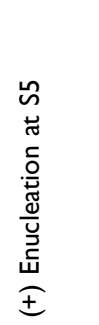 & 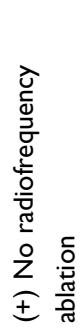 & 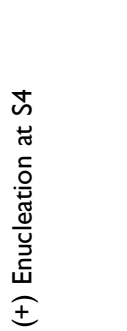 \\
\hline 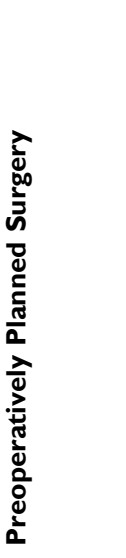 & & 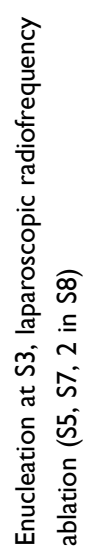 & & 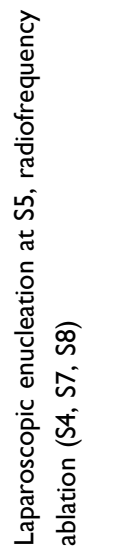 & 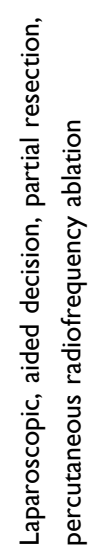 & & 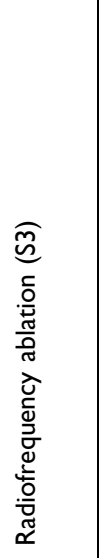 & & 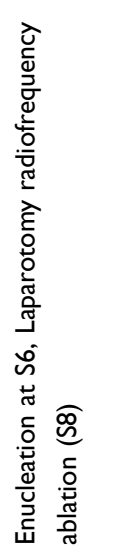 & 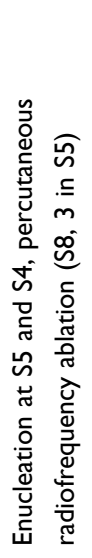 & 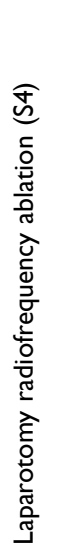 & 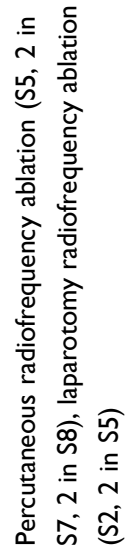 \\
\hline 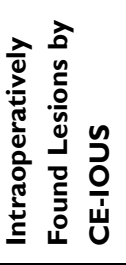 & & 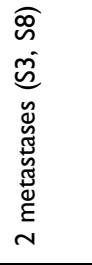 & & 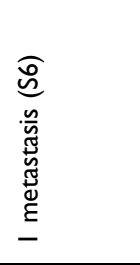 & 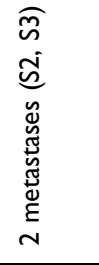 & & $\begin{array}{l}\widehat{\hat{\tilde{g}}} \\
\underline{\mathrm{n}} \\
\underline{-} \\
\end{array}$ & & 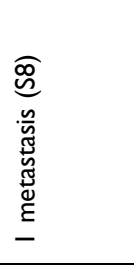 & 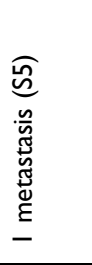 & 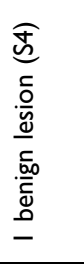 & 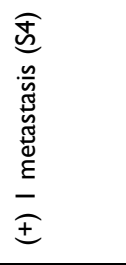 \\
\hline つֶّ & & 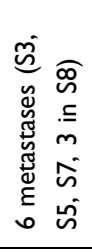 & $\begin{array}{l}\mathbb{E} \\
\frac{\pi}{T} \\
\underline{Z} \\
- \\
\end{array}$ & 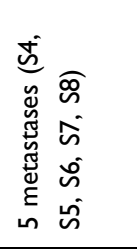 & 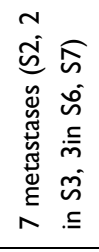 & 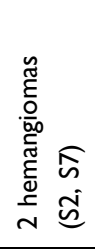 & $\begin{array}{l}\widehat{\hat{n}} \\
\underline{\tilde{n}} \\
- \\
-\end{array}$ & 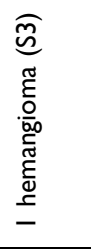 & 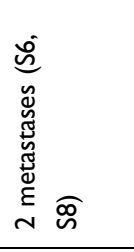 & 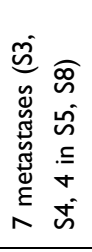 & 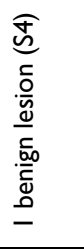 & 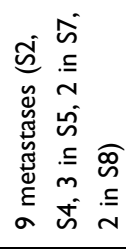 \\
\hline 气 & & 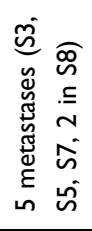 & 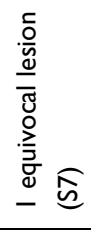 & 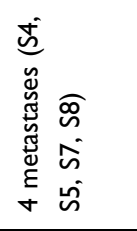 & 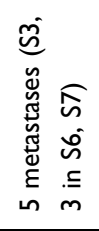 & 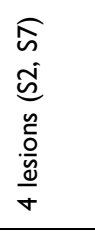 & $\begin{array}{l}\widehat{\hat{\tilde{g}}} \\
\underline{\mathrm{n}} \\
\underline{-}\end{array}$ & 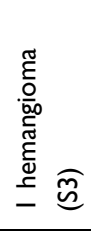 & 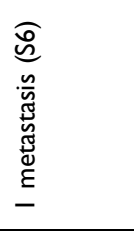 & 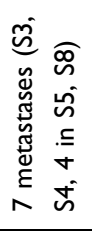 & 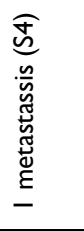 & 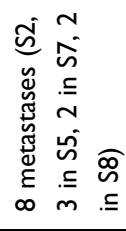 \\
\hline 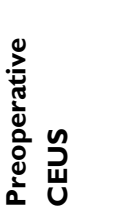 & & 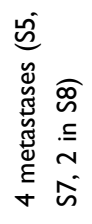 & $\begin{array}{l}\frac{\sigma}{6} \\
\frac{T}{Z} \\
\underline{u} \\
-\end{array}$ & 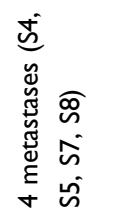 & 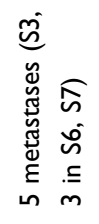 & 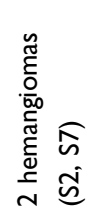 & & 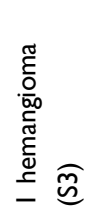 & 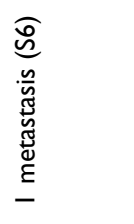 & 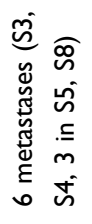 & 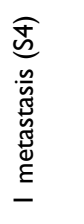 & 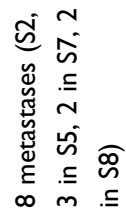 \\
\hline 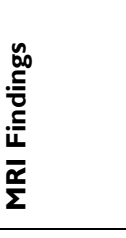 & 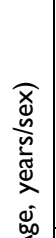 & 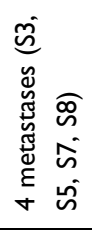 & 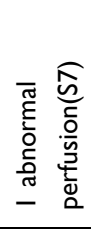 & 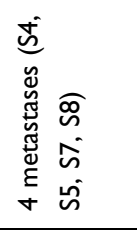 & 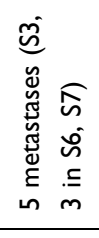 & 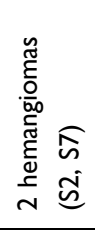 & 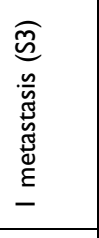 & 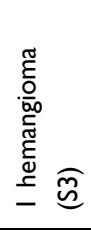 & 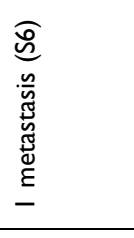 & 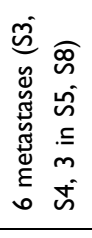 & 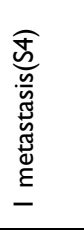 & 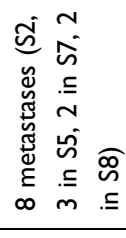 \\
\hline 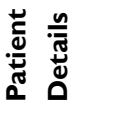 & 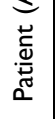 & 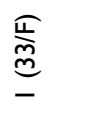 & & 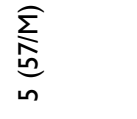 & $\underset{0}{\substack{0 \\
0}}$ & & $\begin{array}{l}\stackrel{\grave{0}}{\underline{\Xi}} \\
\stackrel{\Xi}{\Sigma}\end{array}$ & & $\begin{array}{l}\overline{\bar{\theta}} \\
\underline{\underline{\Sigma}}\end{array}$ & $\begin{array}{l}\stackrel{\bar{\partial}}{\tilde{N}} \widehat{\Sigma} \\
\tilde{\Sigma}\end{array}$ & & 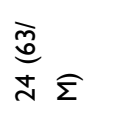 \\
\hline
\end{tabular}


A

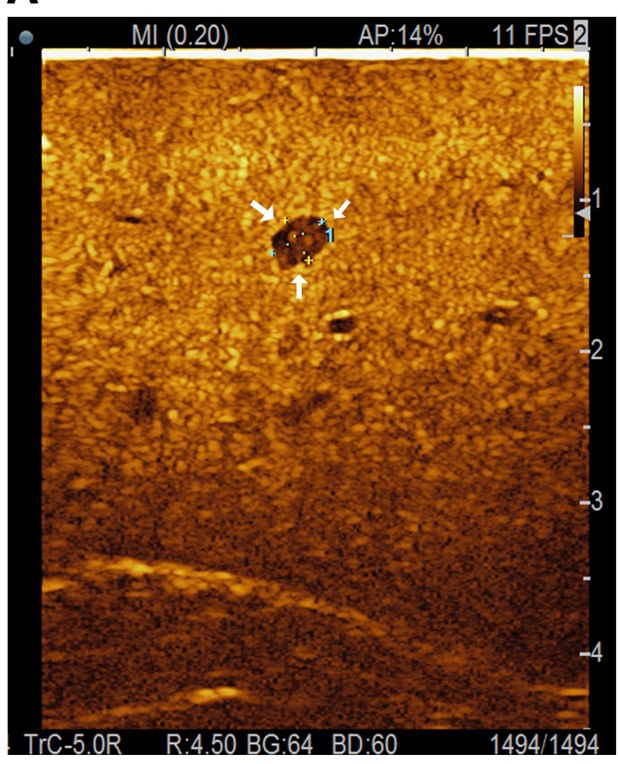

C

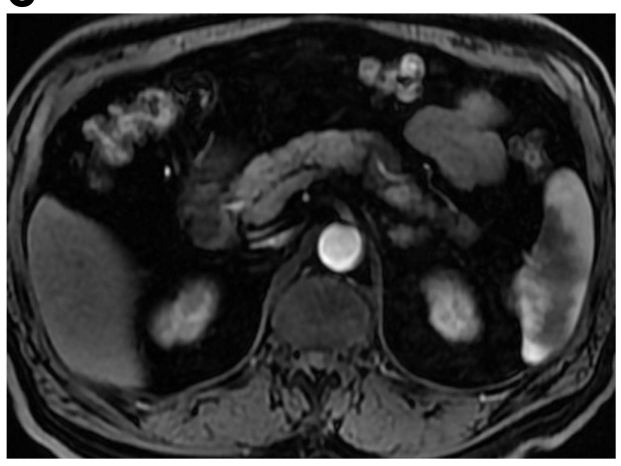

B

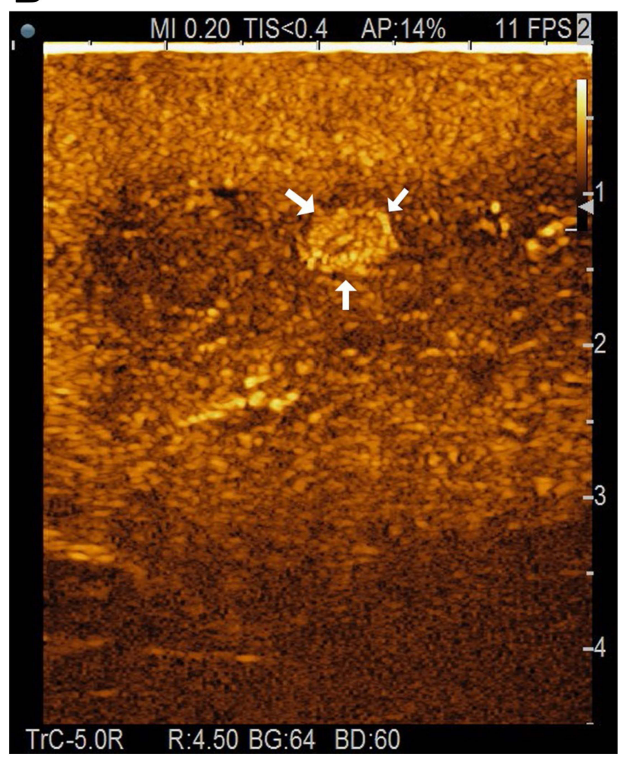

D

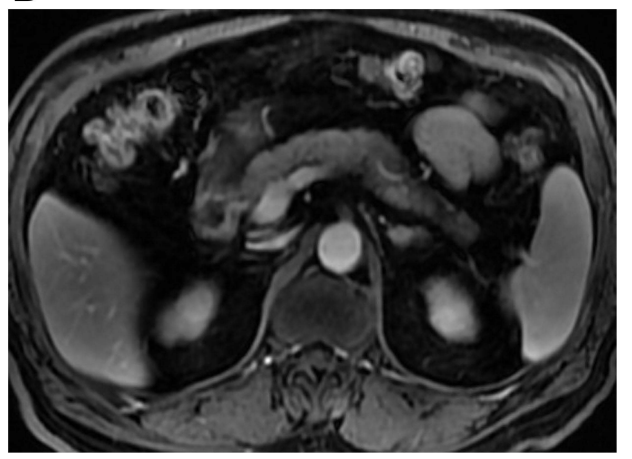

Figure I An occult metastasis. (A) An occult metastasis at the Segment 6 only detected by CE-IOUS. A clear hypo-enhanced lesion (approximately $4 \mathrm{~mm}$ in diameter, white arrow) was newly detected at the Kupffer phase. (B) CE-IOUS in arterial phase (30 seconds) showed hyper-enhancing lesion (white arrow). (C and D) This metastasis could not be found by dynamic MRI.

microbubbles of Sonazoid were taken up by Kupffer cells and sustained in hepatic parenchyma within Kupffer cells lasting up to 60 minutes. The Kupffer phase image shows hyperenhancement in the liver parenchyma. ${ }^{20}$ However, most tumors, especially the malignant tumors, defect Kupffer cells and depict them as hypo-enhancement or absent enhancement. Kupffer phase provides enough time for the whole liver as long as 1 hour, and small occult lesions can be detected which may not be visible with noncontrast imaging, while SonoVue probably does not provide enough time to detect occult lesions that are not visible with non-contrast imaging. On the other hand, other kinds of contrast mediums such as SonoVue, which cannot be devoured by Kupffer cells, provide parenchyma contrast through transient slowdown of microbubbles, and the Kupffer cells hardly phagocytosed the microbubbles of
SonVue. ${ }^{21,22}$ For CE-IOUS examination, SonoVue needs repeated injections to perform a whole-liver examination due to short duration. ${ }^{6,7}$ Meanwhile, CE-IOUS using Sonazoid could precisely investigate a whole liver aimed at detecting the possible metastases. Therefore, Sonazoid seems to be a better choice for CE-IOUS in patients with met.

Lesion-by-lesion analysis in all patients showed that in the assessment of met, CE-IOUS had the highest sensitivity and specificity, albeit not statistically better than other imaging methods. However, CE-IOUS can only be performed during the operation, we believe that CEUS shows the best sensitivity (97.1\%) in the detection of met in patients. On the other hand, CEUS performed preoperatively could not detect extra-hepatic disease comprehensively. The MRI with a sensitivity of $93 \%$ and an accuracy 
of $89 \%$ seems to be the reasonable choice for staging patients towards surgery because it assesses extrahepatic disease as well as intrahepatic metastases. ${ }^{23}$

Limitations of this study should be acknowledged. The main limitation is the small sample. Although there were only 27 patients, we evaluated 99 lesions, which were not too small sample. However, we still found this interesting result in such a small sample, and we thought that we could share this experience with others because patients would benefit from this new technique, and we need further multi-center studies with larger sample to confirm the findings of this study in the future.

\section{Conclusions}

CE-IOUS may play a similar or even better role than other radiological methods in diagnosing liver metastasis. The CE-IOUS using Sonazoid demonstrated a high sensitivity and specificity for finding occult metastases intraoperatively and changing the treatment strategy.

\section{Disclosure}

The authors declare no conflicts of interest for this work.

\section{References}

1. Torzilli G, Montorsi M, Donadon M, et al. "Radical but conservative" is the main goal for ultrasonography-guided liver resection: prospective validation of this approach. $J$ Am Coll Surg. 2005;201 (4):517-528. doi:10.1016/j.jamcollsurg.2005.04.026

2. Jarnagin WR, Bach AM, Winston CB, et al. What is the yield of intraoperative ultrasonography during partial hepatectomy for malignant disease? J Am Coll Surg. 2001;192(5):577-583. doi:10.1016/ s1072-7515(01)00794-3

3. Agrawal N, Fowler AL, Thomas MG. The routine use of intraoperative ultrasound in patients with colorectal cancer improves the detection of hepatic metastases. Colorectal Dis. 2006;8(3):192-194. doi:10.1111/j.1463-1318.2005.00916.x

4. Charnley RM, Morris DL, Dennison AR, Amar SS, Hardcastle JD. Detection of colorectal liver metastases using intraoperative ultrasonography. $\quad B r \quad J \quad$ Surg. 1991;78(1):45-48. doi:10.1002/ bjs. 1800780116

5. Mazzoni G, Napoli A, Mandetta S, et al. Intra-operative ultrasound for detection of liver metastases from colorectal cancer. Liver Int. 2008;28 (1):88-94. doi:10.1111/j.1478-3231.2007.01583.x

6. Torzilli G, Del Fabbro D, Palmisano A, et al. Contrast-enhanced intraoperative ultrasonography during hepatectomies for colorectal cancer liver metastases. J Gastrointest Surg. 2005;9(8):1148-1153. doi:10.1016/j.gassur.2005.08.016

7. Leen E, Ceccotti P, Moug SJ, et al. Potential value of contrast-enhanced intraoperative ultrasonography during partial hepatectomy for metastases: an essential investigation before resection? Ann Surg. 2006;243(2):236-240. doi:10.1097/01. sla.0000197708.77063.07

8. Kudo M. Defect reperfusion imaging with Sonazoid $^{\mathbb{B}}$ : a breakthrough in hepatocellular carcinoma. Liver Cancer. 2016;5(1):1-7. doi: $10.1159 / 000367760$
9. Kim TK, Jang HJ. Contrast-enhanced ultrasound in the diagnosis of nodules in liver cirrhosis. World $J$ Gastroenterol. 2014;20 (13):3590-3596. doi:10.3748/wjg.v20.i13.3590

10. D’Onofrio M, Crosara S, De Robertis R, et al. Contrast-enhanced ultrasound of focal liver lesions. AJR Am J Roentgenol. 2015;205(1): W56-66. doi:10.2214/AJR.14.14203

11. Kudo M, Hatanaka K, Maekawa K. Newly developed novel ultrasound technique, defect reperfusion ultrasound imaging, using sonazoid in the management of hepatocellular carcinoma. Oncology. 2010;78(Suppl 1):40-45. doi:10.1159/000315229

12. Hatanaka K, Kudo M, Minami Y, et al. Differential diagnosis of hepatic tumors: value of contrast-enhanced harmonic sonography using the newly developed contrast agent, Sonazoid. Intervirology. 2008;51(Suppl 1):61-69. doi:10.1159/000122600

13. Mitsunori Y, Tanaka S, Nakamura N, et al. Contrast-enhanced intraoperative ultrasound for hepatocellular carcinoma: high sensitivity of diagnosis and therapeutic impact. $J$ Hepatobiliary Pancreat Sci. 2013;20(2):234-242. doi:10.1007/s00534-0120507-9

14. Mishima M, Toh U, Iwakuma N, Takenaka M, Furukawa M, Akagi Y. Evaluation of contrast Sonazoid-enhanced ultrasonography for the detection of hepatic metastases in breast cancer. Breast Cancer. 2016;23(2):231-241. doi:10.1007/s12282-014-0560-0

15. Nakano H, Ishida Y, Hatakeyama T, et al. Contrast-enhanced intraoperative ultrasonography equipped with late Kupffer-phase image obtained by sonazoid in patients with colorectal liver metastases. World J Gastroenterol. 2008;14(20):3207-3211. doi:10.3748/ wjg.14.3207

16. Shah AJ, Callaway M, Thomas MG, Finch-Jones MD. Contrastenhanced intraoperative ultrasound improves detection of liver metastases during surgery for primary colorectal cancer. HPB (Oxford). 2010;12(3):181-187. doi:10.1111/j.14772574.2009.00141.x

17. Liu W, Zhang ZY, Yin SS, Yan K, Xing BC. Contrast-enhanced intraoperative ultrasound improved sensitivity and positive predictive value in colorectal liver metastasis: a systematic review and Meta-analysis. Ann Surg Oncol. 2020;2:254. doi:10.1245/s10434020-09365-X

18. Oba $\mathrm{A}$, Mise $\mathrm{Y}$, Ito $\mathrm{H}$, et al. Clinical implications of disappearing colorectal liver metastases have changed in the era of hepatocyte-specific MRI and contrast-enhanced intraoperative ultrasonography. $H P B(O x f o r d)$. 2018;20(8):708-714. doi:10.1016/j. hpb.2018.02.377

19. Yao J, Teupe C, Takeuchi M, et al. Quantitative 3-dimensional contrast echocardiographic determination of myocardial mass at risk and residual infarct mass after reperfusion: experimental canine studies with intravenous contrast agent NC100100. J Am Soc Echocardiogr. 2000;13(6):570-581. doi:10.1067/mje.2000.104646

20. Watanabe R, Matsumura M, Munemasa T, Fujimaki M, Suematsu M. Mechanism of hepatic parenchyma specific contrast of microbubble-based contrast agent for ultrasonography: microscopic studies in rat liver. Invest Radiol. 2007;42(9):643-651. doi:10.1097/ RLI.0b013e1805f 2682

21. Yanagisawa $\mathrm{K}$, Moriyasu $\mathrm{F}$, Miyahara $\mathrm{T}$, Yuki M, Iijima $\mathrm{H}$. Phagocytosis of ultrasound contrast agent microbubbles by Kupffer cells. Ultrasound Med Biol. 2007;33(2):318-325. doi:10.1016/j. ultrasmedbio.2006.08.008

22. Kono Y, Steinbach GC, Peterson T, Schmid-Schonbein GW, Mattrey RF. Mechanism of parenchymal enhancement of the liver with a microbubble-based US contrast medium: an intravital microscopy study in rats. Radiology. 2002;224(1):253-257. doi:10.1148/ radiol.2241011352

23. Rojas Llimpe FL, Di Fabio F, Ercolani G, et al. Imaging in resectable colorectal liver metastasis patients with or without preoperative chemotherapy: results of the PROMETEO-01 study. $\mathrm{Br} J$ Cancer. 2014;111(4):667-673. doi:10.1038/bjc.2014.351 


\section{Publish your work in this journal}

Therapeutics and Clinical Risk Management is an international, peerreviewed journal of clinical therapeutics and risk management, focusing on concise rapid reporting of clinical studies in all therapeutic areas, outcomes, safety, and programs for the effective, safe, and sustained use of medicines. This journal is indexed on PubMed Central, CAS,
EMBase, Scopus and the Elsevier Bibliographic databases. The manuscript management system is completely online and includes a very quick and fair peer-review system, which is all easy to use. Visit http://www.dovepress.com/testimonials.php to read real quotes from published authors.

Submit your manuscript here: https://www.dovepress.com/therapeutics-and-clinical-risk-management-journal 